\title{
Factores de riesgo asociados a bacteriemias en niños quemados internados en una unidad de cuidados intensivos pediátricos especializada: estudio de casos y controles \\ Risk factors associated with bacteremia in burn children \\ admitted to a specialized pediatric intensive care unit: \\ A case-control study
}

\author{
Dr. Hugo Basilico ${ }^{a}$, Dr. Sebastián García y Dr. Lucas Pintos ${ }^{a}$
}

\section{RESUMEN}

Introducción. Las infecciones con bacteriemias en pacientes quemados son causa frecuente de complicaciones y aumento de días de internación. El conocimiento de los microorganismos causales y la identificación de factores de riesgo asociados permiten disminuir las complicaciones infecciosas, la morbimortalidad y los costos en cuidados de la salud. Este trabajo evalúa el grado de asociación entre los factores de riesgo y los episodios de bacteriemias en pacientes quemados, e identifica los microorganismos aislados más frecuentemente en hemocultivos. Población y métodos. Estudio de casos y controles realizado en la Unidad de Cuidados Críticos de Quemados del Hospital de Pediatría S.A.M.I.C. "Prof. Dr. Juan P. Garrahan" entre el 1 de junio de 2014 y el 30 de septiembre de 2019 en pacientes que presentaron episodios de bacteriemia con hemocultivo positivo (casos) y los que presentaron hemocultivos negativos (controles).

Resultados. Durante el período estudiado se identificaron 29 casos de bacteriemias. La mediana de días de internación al momento del episodio de bacteriemia fue de 23 días. El microorganismo más frecuentemente identificado fue Pseudomonas (7 casos). El único factor de riesgo con el que se pudo establecer asociación significativa fue la presencia de acceso venoso central con 7 días o más (OR 3,18; IC $95 \%$ : 1,20-8,38). La mortalidad global fue del $9,1 \%$, en los casos fue del $13,8 \%$, y en los controles, del 3,4\%.

Conclusiones. Los accesos venosos centrales con duración mayor a 7 días son un factor de riesgo independiente de bacteriemias en niños quemados críticos. No se pudo establecer una asociación estadísticamente significativa con otros factores de riesgos analizados.Pseudomonas, Acinetobacter y Staphylococcus coagulasa negativo fueron los microorganismos más frecuentemente identificados en las bacteriemias.

Palabras clave: bacteriemia, quemaduras, infecciones, morbimortalidad.

http:/ / dx.doi.org/10.5546/ aap.2021.325

Texto completo en inglés:

http:/ / dx.doi.org/10.5546/ aap.2021.eng.325
Cómo citar: Basílico H, García S, Pintos L. Factores de riesgo asociados a bacteriemias en niños quemados internados en una unidad de cuidados intensivos pediátricos especializada: estudio de casos y controles. Arch Argent Pediatr 2021;119(5):325-330.

\section{INTRODUCCIÓN}

Aproximadamente el $75 \%$ de los pacientes pediátricos que sufren quemaduras son menores de 5 años y el principal sitio donde ocurren las lesiones es en el hogar. La etiología más frecuente son los líquidos calientes en niños menores de 4 años y el fuego directo en los mayores. ${ }^{1}$

Las infecciones son una causa de complicaciones y aumento de los días de internación. ${ }^{2}$ Además, continúan siendo una causa significativa de morbimortalidad ya que, aunque la mortalidad ha declinado como consecuencia de los avances en el manejo clínico-quirúrgico y la prevención de complicaciones, la mortalidad secundaria a infección a punto de partida de la quemadura es aún muy elevada ${ }^{2,3}$ y constituye la causa principal de mortalidad en pacientes quemados. ${ }^{4}$

Las infecciones son frecuentes debido al compromiso de la barrera cutánea, la destrucción de la flora residente y la alteración de la inmunidad que generan condiciones favorables para la colonización bacteriana y el desarrollo de microorganismos resistentes al tratamiento. ${ }^{5}$ Los pacientes se colonizan por microorganismos endógenos procedentes de la 
piel, del tracto gastrointestinal o de la vía respiratoria. En los pacientes hospitalizados, la colonización puede adquirirse del ambiente hospitalario y puede involucrar microorganismos multirresistentes. Staphylococcus aureus, Pseudomonas aeruginosa y especies de enterobacterias son los microorganismos más frecuentes. ${ }^{6-8}$ Las bacteriemias suelen producirse por invasión de microorganismos de la piel, aunque también pueden originarse al momento de desbridación quirúrgica, por colonización de accesos venosos centrales, infección urinaria o translocación bacteriana del tracto respiratorio. ${ }^{9}$ Los criterios de la American Burn Association (ABA) para el diagnóstico de sepsis ( 3 criterios o más de los siguientes: temperatura $>39^{\circ} \mathrm{Co}<36,5^{\circ} \mathrm{C}$, taquicardia progresiva, taquipnea progresiva, plaquetopenia, hiperglucemia e intolerancia a la vía oral) tienen una correlación limitada con la presencia de bacteriemia confirmada con hemocultivos, con una sensibilidad del 78,2 \% y una especificidad del 49,5\%.10,11

El objetivo fue evaluar el grado de asociación entre los factores de riesgo y los episodios de bacteriemias en pacientes quemados, e identificar los microorganismos aislados más frecuentemente en hemocultivos y sus respectivas sensibilidades antibióticas.

\section{POBLACIÓN, MATERIALES Y MÉTODOS}

Estudio realizado en la Unidad de Cuidados Críticos de Quemados del Hospital de Pediatría S.A.M.I.C. "Prof. Dr. Juan P. Garrahan" de la Ciudad Autónoma de Buenos Aires, entre el 1 de junio de 2014 y el 30 de septiembre de 2019.

Diseño: Estudio de casos y controles prevalentes. Los datos fueron obtenidos retrospectivamente de las historias clínicas informatizadas.

\section{Criterios de inclusión}

- Casos: pacientes de 0 a 17 años y 11 meses que durante la internación presentaron episodios de bacteriemia (cultivo positivo en sangre) durante el período de estudio.

- Controles: pacientes de 0 a 17 años y 11 meses internados en UCIP en el mismo período de tiempo con hemocultivos tomados por sospecha de bacteriemia que fueron negativos. Se obtuvieron los datos de las historias clínicas informatizadas del total de los pacientes que presentaron bacteriemias informados por el Servicio de Epidemiología e Infectología y se seleccionaron al azar entre los pacientes con hemocultivos negativos las historias clínicas de los controles con una relación caso:control 1:2.

\section{Criterios de exclusión}

Episodio de bacteriemia constatado en otra institución; desconocimiento de días de colocación del acceso venoso o desconocimiento respecto a ingreso a quirófano 24 horas previas en pacientes derivados de otras instituciones.

\section{Descripción de variables}

- Variable dependiente (bacteriemia): presencia de microorganismo aislado en cultivo de sangre dentro de los 5 días de extraída la muestra.

- Variables independientes (de exposición): edad en meses, superficie corporal quemada (porcentaje de piel afectada), tiempo de internación: en días desde el ingreso a nuestra institución, sonda vesical (presencia o no), tiempo y localización de acceso venoso central (menos de 7 días y más de 7 días, y sitio de inserción anatómico), asistencia respiratoria mecánica (ARM) (días de evolución de asistencia respiratoria), intervención quirúrgica mediata (ingreso a quirófano 24 horas previas a episodios de bacteriemia).

Análisis estadístico: Se obtuvieron los datos de las historias clínicas informatizadas de los pacientes que presentaron bacteriemias reportados por el Servicio de Epidemiología e Infectología y se seleccionaron al azar las historias clínicas de los controles. Se utilizó para la comparación de variables cualitativas la prueba de chi cuadrado, las variables cuantitativas de distribución normal fueron comparadas con la prueba $T$ de Student y las de distribución asimétrica con la prueba de Wilcoxon. Se calcularon los odds ratio (OR) para evaluar el grado de asociación con los factores de riesgo. Para el procesamiento de los datos se utilizó el software InfoStat versión $2020^{\circledR}$ (Universidad Nacional de Córdoba) y Epidat versión $4.1^{\circledR}$ (Organización Panamericana de la Salud).

Control de sesgo: Se identificaron todos los casos de bacteriemias informados en planillas de control epidemiológico y se seleccionaron aleatoriamente los controles en cumplimento de los criterios de inclusión y exclusión de ambos grupos antes mencionados.

Aspectos éticos: El presente trabajo dio cumplimiento a todas las leyes y a la reglamentación vigente respecto a pautas de 
evaluación ética, y fue aprobado por el Comité de Investigación de la institución. La recopilación de datos se realizó con identificaciones cifradas protegiendo la identidad de los pacientes. Debido al carácter observacional y retrospectivo, no fue necesaria la realización de consentimientos informados.

\section{RESULTADOS}

Durante el período estudiado se internaron 230 pacientes quemados, $61 \%$ de sexo masculino; la mediana de la edad de la población estudiada fue de 32 meses con rango entre 2 meses y 14 años. En la Tabla 1 se muestran las características descriptivas de los casos y los controles. Se diagnosticaron 29 episodios de bacteriemias en 16 pacientes $(6,96 \%)$ y se seleccionaron 58 controles.

En los pacientes con edad menor a 12 meses, se identificaron 5 casos de bacteriemias $(17,8 \%)$ y 6 controles $(10,2 \%)$, sin poder establecerse asociación estadísticamente significativa.

El porcentaje de superficie corporal quemada (SCQ) osciló entre el $15 \%$ y el $87 \%$, con un promedio del $50 \%$ con una desviación estándar (DE) de 20,7 y sin diferencias estadísticas respecto al número de bacteriemias en el corte del $30 \%$ de SCQ.

TABla 1. Características de casos y controles

\begin{tabular}{lccc}
\hline Características & $\begin{array}{c}\text { Casos } \\
(\mathbf{n}=\mathbf{2 9})\end{array}$ & $\begin{array}{c}\text { Controles } \\
(\mathbf{n}=\mathbf{5 8})\end{array}$ & Valor de $p$ \\
\hline Edad (meses) & 32 & 113 & 0,01 \\
Quemadura (\%) & 50 & 35 & 0,15 \\
ARM (días) & 21 & 14 & 0,11 \\
Internación (días) & 23 & 16 & 0,12 \\
Sonda vesical (\%) & 90 & 78 & 0,16 \\
Mortalidad (\%) & 13,8 & 3,4 & 0,003 \\
Ingreso al quirófano (\%) & 51 & 62 & 0,35 \\
\hline
\end{tabular}

ARM: asistencia respiratoria mecánica.
La mediana de días de internación al momento del episodio de bacteriemia fue de 23 días, en tanto que la mediana de evolución con ventilación mecánica (ARM) fue de 21 días. Ambas variables sin asociación estadísticamente significativas.

La mortalidad global en pacientes internados durante dicho período fue del 9,1\%; en los casos fue del 13,8 \% y en los controles del 3,4 \% $(p=0,003)$.

Se identificaron 27 episodios de bacteriemias en pacientes con presencia de acceso venoso central: 16 con una duración de $>7$ días de colocado el acceso y 11 con $\leq 7$ días. El análisis estadístico asocia la permanencia del acceso venoso central mayor a 7 días como un factor de riesgo de bacteriemia ( $p=0,017$; IC $95 \%$ : 3,18$8,38)$. La localización del acceso venoso más frecuentemente utilizada fue femoral $(74 \%)$, seguida de yugular $(18,5 \%)$ y subclavia $(7,5 \%)$; no se pudo establecer asociación entre localización y mayor riesgo de infecciones (Tabla 2).

El abordaje quirúrgico fue planteado como un factor de riesgo y se identificaron los pacientes que presentaron bacteriemias dentro de las 24 horas posteriores a su ingreso, no se estableció una asociación estadísticamente significativa al igual que lo ocurrido con la utilización de sonda vesical (Tabla 3).

Los microorganismos más frecuentemente aislados fueron $P$. aureginosa (7 casos), Acinetobacter spp. (5 casos) y Staphylococcus coagulasa negativo (4 casos). En la Tabla 4 se muestra el aislamiento microbiológico según germen y localización; el $S$. aureus se rescató solamente en 3 casos. En cuanto a la resistencia en función de los antibióticos utilizados como terapia empírica inicial ante sospecha de sepsis o descompensación, se consideró la resistencia al meropenem en microorganismos gramnegativos y a la meticilina en microorganismos grampositivos,

TABLA 2. Característica de accesos venosos centrales

\begin{tabular}{|c|c|c|c|c|}
\hline & Casos & Controles & Valor de $p$ & OR (IC $95 \%$ ) \\
\hline \multicolumn{5}{|l|}{ Duración } \\
\hline > 7 días & 16 & 16 & \multirow{2}{*}{0,017} & \multirow{2}{*}{$3,18(1,2-8,4)$} \\
\hline$\leq 7$ días & 11 & 35 & & \\
\hline \multicolumn{5}{|l|}{ Localización } \\
\hline Femoral & 20 & 38 & 0,36 & $2,10(0,4-10,9)$ \\
\hline Yugular & 5 & 7 & 0,27 & $2,85(0,4-19,6)$ \\
\hline Subclavia & 2 & 6 & 0,79 & $1,33(0,14-2,4)$ \\
\hline
\end{tabular}

OR: odds ratio, IC 95\%: intervalo de confianza del $95 \%$. 
observándose en un único paciente $P$. aeruginosa resistente a meropenem y un único caso de S. aureus meticilino resistente (SAMR).

\section{DISCUSIÓN}

El mejor conocimiento sobre los factores de riesgo, la etiología y la sensibilidad antibiótica en bacteriemias de pacientes quemados críticos permite disminuir la morbimortalidad. En la población analizada, la superficie corporal quemada osciló entre un $15 \%$ y un $87 \%$, con un promedio del $50 \%$, lo que la convertía en una población críticamente enferma, susceptible de múltiples factores de riesgo de infecciones.
Considerando la gravedad de los cuadros de bacteriemia en niños quemados y visto que los aislamientos microbiológicos son variables según cada institución, es necesario conocer la epidemiología local de cada centro. En nuestro hospital, los gérmenes más frecuentemente aislados (P. aeruginosa, Acinetobacter y S. aureus) representan aproximadamente el $50 \%$, en coincidencia con los informes publicados por los distintos centros especializados. ${ }^{8,9,12-14}$ En un estudio realizado por Fochtmann-Frana et al., las infecciones del torrente sanguíneo fueron causadas por bacterias gramnegativas $(46 \%)$, bacterias grampositivas (40\%) y hongos $(14 \%)$.

TABLA 3. Análisis bivariado de factores de riesgo

\begin{tabular}{|c|c|c|c|c|c|c|}
\hline & & Casos & Controles & Total & Valor de $p$ & OR (IC95\%) \\
\hline \multirow[t]{2}{*}{ Edad } & $\leq 12$ meses & 6 & 5 & 11 & \multirow{2}{*}{0,11} & \multirow{2}{*}{$2,76(0,77-9,98)$} \\
\hline & $>12$ meses & 23 & 53 & 76 & & \\
\hline \multirow[t]{2}{*}{ Ingreso al quirófano } & Sí & 15 & 36 & 51 & \multirow{2}{*}{0,36} & \multirow[t]{2}{*}{$0,65(0,27-1,61)$} \\
\hline & No & 14 & 22 & 36 & & \\
\hline \multirow[t]{2}{*}{ Sonda vesical } & Sí & 26 & 45 & 71 & \multirow{2}{*}{0,17} & \multirow{2}{*}{$2,5(0,65-9,61)$} \\
\hline & No & 3 & 13 & 16 & & \\
\hline \multirow[t]{2}{*}{ Días de internación } & > 14 días & 19 & 35 & 54 & \multirow{2}{*}{0,64} & \multirow{2}{*}{$1,25(0,49-3,16)$} \\
\hline & $\leq 14$ días & 10 & 23 & 33 & & \\
\hline \multirow[t]{2}{*}{ ARM } & > 14 días & 14 & 21 & 35 & \multirow[t]{2}{*}{0,27} & \multirow[t]{2}{*}{$1,64(0,66-4,06)$} \\
\hline & $\leq 14$ días & 15 & 37 & 52 & & \\
\hline \multirow[t]{2}{*}{ CVC } & $>7$ días & 16 & 16 & 32 & \multirow{2}{*}{0,017} & \multirow{2}{*}{$3,18(1,20-8,38)$} \\
\hline & $<7$ días & 11 & 35 & 46 & & \\
\hline \multirow[t]{2}{*}{ Superficie quemada } & $>30 \%$ & 24 & 46 & 70 & \multirow[t]{2}{*}{0,7} & \multirow[t]{2}{*}{$1,25(0,39-3,97)$} \\
\hline & $\leq 30 \%$ & 5 & 12 & 17 & & \\
\hline
\end{tabular}

ARM: asistencia respiratoria mecánica, CVC: catéter venoso central, OR: odds ratio, IC $95 \%$ : intervalo de confianza del $95 \%$.

TABLA 4. Aislamiento microbiológico según microorganismo y localización

\begin{tabular}{lcccc}
\hline Microorganismo & Hemocultivos $(\mathrm{n}=29)$ & Cultivo de piel $(\mathrm{n}=13)$ & Punta de catéter $(\mathrm{n}=12)$ & Urocultivos $(\mathrm{n}=5)$ \\
\hline Pseudomonas & 7 & 4 & 3 & 2 \\
Acinetobacter & 5 & - & 3 & 1 \\
S. coagulasa neg. & 4 & 4 & 2 & - \\
S. aureus & 3 & 3 & - & - \\
Enterococo & 2 & - & 1 & 1 \\
Klebsiella & 2 & - & - & - \\
Serratia & 2 & - & - & - \\
Stenotrophomona & 2 & 1 & 1 & - \\
Providencia & 1 & - & 1 & 1 \\
Candida & 1 & 1 & 1 & - \\
\hline
\end{tabular}


Los patógenos más comunes aislados fueron Enterococcus, Candida, Pseudomonas, Enterobacter, S. aureus, estafilococos coagulasa negativos y Klebsiella. ${ }^{12}$ En el año 2017, en un centro de referencia de Colombia, Ramirez-Blanco et al. notificaron los gérmenes más frecuentemente aislados: P. aeruginosa, A. baumannii, Escherichia coli, S. aureus y Klebsiella pneumoniae; ${ }^{15}$ y se observaron resultados similares a los de en nuestro trabajo. No obstante, otros estudios identifican las bacterias grampositivas como la causa más común de bacteriemia en pacientes con quemaduras $(66,4 \%)$, seguidas de bacterias gramnegativas $(22,1 \%)$ y hongos $(11,5 \%)$, sobre todo durante la primera semana de internación. ${ }^{7}$

Ante la descompensación clínica de un paciente gravemente quemado, se debe tener la presunción de una complicación infecciosa y proceder a realizar cultivos, e iniciar o ampliar el esquema antibiótico. ${ }^{16} \mathrm{El}$ tratamiento empírico inicial inadecuado puede aumentar la mortalidad. ${ }^{17,18}$ Devrim et al., en un estudio, evidenciaron 8 de 9 casos de bacteriemia a $P$. aeruginosa resistente a carbapenémicos; 1 aislamiento de K. pneumoniae $(12,5 \%)$ y 2 aislamientos de E. coli $(22,2 \%)$ betalactamasas de espectro extendido (BLEE) positivos y 27 de 70 casos de resistencia a la meticilina. ${ }^{7}$ En nuestro trabajo no se observaron altas tasas de resistencia para los antibióticos utilizados como terapia empírica inicial (meropenem-vancomicina) ante la descompensación clínica.

El recambio sistemático del acceso venoso central es una recomendación controversial, aunque la permanencia por tiempo prolongado está identificada como un factor de riesgo reversible. ${ }^{10,12,19}$ En nuestra institución, la recomendación es colocar un acceso venoso nuevo al 7. ${ }^{\circ}$ día. Fochtmann-Frana et al. comprobaron la colonización de la punta del catéter venoso central (CVC) en 200 de 472 pacientes (42\%) después de una media de 12 días, por lo que consideramos lógico, oportuno y prudente el recambio de CVC todas las semanas, ${ }^{12}$ de manera de disminuir el riesgo evidenciado en nuestro estudio (OR 3,18; IC 95\%: 1,20-8,38). No hay datos publicados en los últimos años respecto al punto de corte para realizar el recambio sistemático preventivo de accesos venosos centrales.

El 13,8 \% de los pacientes que presentaron bacteriemias fallecieron, frente al 3,4 \% de los fallecidos en el grupo control. Si bien es multicausal el desenlace fatal, ${ }^{20,21}$ aquellos pacientes con mayor superficie corporal quemada, infecciones asociadas ${ }^{22}$ y mayor duración de la internación presentan mayores tasas de mortalidad..$^{14}$ Es evidente que el elevado número de bacteriemias en los pacientes quemados representa un gran problema en las instituciones especializadas debido a su alta morbimortalidad, aumento de costos y días de internación. Los equipos de salud tienen grandes desafíos por delante para disminuir la morbimortalidad: conocer la epidemiología local, protocolizar el tratamiento antibiótico empírico inicial y establecer conjuntos de medidas (bundles) para la colocación y mantenimiento de accesos venosos centrales. ${ }^{23-25}$

Cabe mencionar que la principal limitación del estudio fue la baja prevalencia de episodios de bacteriemias en el tiempo examinado, que no permitió establecer asociaciones estadísticamente significativas. El diseño de un estudio de tipo prospectivo, con un tamaño muestral mayor, donde se puedan evaluar otros factores y variables que influyen en episodios de bacteriemias, permitirá obtener información estadísticamente significativa y extrapolar los datos a la población susceptible.

\section{CONCLUSIÓN}

En nuestro estudio la permanencia de acceso venoso central durante más de 7 días tuvo una asociación estadísticamente significativa con episodios de bacteriemia; no fue posible establecer asociación con otros factores de riesgo potenciales descritos en la bibliografía. Pseudomonas, Acinetobacter y S. coagulasa negativo fueron los microorganismos más frecuentemente identificados.

\section{Agradecimiento}

A la Lic. Verónica Álvarez por su colaboración y al Dr. Alberto Belocchio por su indispensable contribución.

\section{REFERENCIAS}

1. Cuenca-Pardo J, Álvarez-Díaz CJ, Comprés-Pichardo TA. Related factors in burn children. Epidemiological study of the Burn Unit at the "Magdalena de las Salinas" Traumatology Hospital. J Burn Care Res. 2008;29(3):468-74.

2. Rosanova MT, Stamboulian D, Lede R. Infecciones en los niños quemados: análisis epidemiológico y de los factores de riesgo. Arch Argent Pediatr. 2013; 111(4):303-8.

3. Rosanova MT, Mudryck G, Villasboas M, Basilico H, et al. Complicaciones infecciosas en pacientes quemados pediátricos. Rev Med Infant. 2009; 16(4):394-9.

4. Sheridan R. Sepsis in pediatric burn patients. Pediatr Crit Care Med. 2005; 6(3 Suppl):S112-9.

5. Ortiz Gil M, Mora Domínguez J, Aguilera Alfonso A. Colonización bacteriana y susceptibilidad antibacteriana de 
Pseudomonas aeruginosa aisladas de pacientes quemados infectados del Hospital Regional de Alta Especialidad de Veracruz. Enf Infec Microbiol. 2009; 29(1):11-9.

6. Chávez-Velarde $\mathrm{T}$, Lona-Reyes J, Riebeling-Dueñas A, Orozco-Alatorre L. Incidencia y factores de riesgo para bacteriemia en pacientes pediátricos con quemaduras en un Centro de Atención Especializado en México. Rev Chil Infectol. 2017; 34(3):221-6.

7. Devrim I, Kara A, Düzgöl M, Karkiner A, et al. Burnassociated bloodstream infections in pediatric burn patients: Time distribution of etiologic agents. Burns. 2017; 43(1):144-8.

8. Rastegar Lari AR, Alaghehbandan R, Akhlaghi L. Burn wound infections and antimicrobial resistance in Tehran, Iran: an increasing problem. Ann Burns Fire Disasters. 2005; 18(2):68-73

9. Rodgers L, Mortensen J, Fisher MC, Lo A, et al. Predictors of infectious complications after burn injuries in children. Pediatr Infect Dis J. 2000; 19(10):990-5.

10. Greenhalgh DG, Saffle JR, Holmes JH $4^{\text {th }}$, Gamelli RL, et al. American Burn Association Consensus Conference to Define Sepsis and Infection in Burns. J Burn Care Res. 2007; 28(6):776-90.

11. Tridente A. Sepsis 3 and the burns patient: do we need Sepsis 3.1? Scars Burns Heal. 2018; 4:2059513118790658.

12. Fochtmann-Frana A, Freystätter C, Vorstandlechner V, Barth A, et al. Incidence of risk factors for bloodstream infections in patients with major burns receiving intensive care: A retrospective single-center cohort study. Burns. 2018; 44(4):784-92.

13. Sabatier C, Peredo R, Vallés J. Bacteriemia en el paciente crítico. Rev Med Intensiva. 2009; 33(7):336-45.

14. Ekrami A, Kalantar E. Bacterial infections in burn patients at a burn hospital in Iran. Indian J Med Res. 2007; 126(6): 541-4.

15. Ramirez-Blanco CE, Ramirez-Rivero CE, Díaz-Martínez LA, Sosa-Ávila LM. Infection in burn patients in a referral center in Colombia. Burns. 2017; 43(3):642-53.

16. Hogan B, Wolf SE, Hospenthal DR, D'Avignon LC, et al. Correlation of American Burn Association sepsis criteria with the presence of bacteremia in burned patients admitted to the intensive care unit. J Burn Care Res. 2012;33(3):371-8.

17. Branski L, Al-Mousawi A, Rivero H, Jeschke MG, et al. Emerging Infections in Burns. Surg Infect (Larchmt). 2009; 10(5):389-97.

18. Núñez López O, Cambiaso-Daniel J, Branski LK, Norbury $\mathrm{WB}$, et al. Predicting and managing sepsis in burn patients: current perspectives. Ther Clin RiskManag. 2017; 13:1107-17.

19. Franceschi D, Gerding R, Phillips G, Fratianne R. Risk factors associated with intravascular catheters infections in burned patients: a prospective, randomized study. J Trauma. 1989; 29(6):811-6.

20. Vostrugina K, GudavièienëD, VitkauskienëA. Bacteremias in patients with severe burn trauma. Medicina (Kaunas). 2006; 42(7):576-9.

21. Balmelli B, Sandoval J, Canata G. Infecciones en niños quemados internados en el Centro Nacional de Quemados y Cirugías Reconstructivas (CENQUER)Paraguay de Enero 2017 a Enero 2018. Rev Salud Pública Parag. 2018; 8(2):45-51.

22. Bang RL, Gang RK, Sanyal SC, Mokaddas E, et al. Burn septicaemia: an analysis of 79 patients. Burns. 1998; 24(4):354-61.

23. Devrim I, Oruç Y, Demirag B, Kara A, et al. Central line bundle for prevention of central line-associated bloodstream infection for totally implantable venous access devices (ports) in pediatric cancer patients. J Vasc Access. 2018; 19(4):358-65.

24. ISBIPracticeGuidelines Committee;Steering Subcommittee; Advisory Subcommittee. ISBI practice guidelines for burn care. Burns. 2016; 42(5):953-1021.

25. Padilla Fortunatti CF. Impact of two bundles on central catheter-related bloodstream infection in critically ill patients. Rev Lat Am Enfermagem. 2017; 25:e2951. 